\title{
Exploratory analyses of the association of MRI with clinical, laboratory and radiographic findings in patients with rheumatoid arthritis
}

\author{
Paul Emery, ${ }^{1}$ Désirée van der Heijde, ${ }^{2}$ Mikkel Østergaard, ${ }^{3}$ Philip G Conaghan, ${ }^{1}$ \\ Mark C Genovese, ${ }^{4}$ Edward C Keystone, ${ }^{5}$ Roy Fleischmann, ${ }^{6}$ Elizabeth C Hsia, ${ }^{7,8}$ \\ Weichun $\mathrm{Xu}^{7}{ }^{7}$ Stephen $\mathrm{Xu}^{7}{ }^{7}$ Mahboob U Rahman ${ }^{9}$
}

${ }^{1}$ University of Leeds and NIHR Leeds Musculoskeletal Biomedical Research Unit, Leeds, UK

2Leiden University Medical Center, Leiden, The Netherlands ${ }^{3}$ Copenhagen University Hospital at Glostrup and Hvidovre,

Copenhagen, Denmark

${ }^{4}$ Stanford University, Palo Alto, California, USA

${ }^{5}$ Rebecca MacDonald Centre for Arthritis and Autoimmune Diseases, Mount Sinai Hospital, University of Toronto, Toronto, Canada

${ }^{6}$ University of Texas Southwest Medical Center, Dallas, Texas, USA

${ }^{7}$ Centocor Research and Development, Inc., Malvern, Pennsylvania, USA 8University of Pennsylvania School of Medicine, Philadelphia, Pennsylvania, USA ${ }^{9}$ Pfizer Inc., Collegeville, Pennsylvania, USA

\section{Correspondence to}

Paul Emery, Academic Section of Musculoskeletal Disease, Leeds Institute of Molecular Medicine, Chapel Allerton Hospital, Chapeltown Road, Leeds LS7 4SA, UK p.emery@leeds.ac.uk.

Accepted 17 July 2011 Published Online First 16 September 2011

\section{ABSTRACT}

Objectives Evaluate relationships between MRI and clinical/laboratory/radiographic findings in rheumatoid arthritis (RA).

Methods 637 methotrexate-naive patients (GO-BEFORE) and 444 patients with active RA despite methotrexate (GO-FORWARD) were randomly assigned to subcutaneous placebo + methotrexate, golimumab $100 \mathrm{mg}+$ placebo, golimumab 50mg + methotrexate, or golimumab $100 \mathrm{mg}+$ methotrexate every-4-weeks. In GO-BEFORE $(n=318)$ and GO-FORWARD $(n=240)$ substudies, MRI of dominant wrist/metacarpophalangeal joints were scored for synovitis, bone oedema and bone erosion (RA MRI scoring (RAMRIS) system). Relationships between RAMRIS scores and serum C-reactive protein (CRP), 28-joint count disease activity score (DAS28-CRP) and van der Heijde modified Sharp (vdH-S) scores were assessed.

Results Baseline and weeks 24/28 DAS28-CRP, CRP, and $v d H-S$ generally correlated well with baseline and week 24 RAMRIS synovitis, oedema and erosion scores. Early (week 4) CRP changes correlated with later (week 12) RAMRIS synovitis/oedema change scores; earlier (week 12) changes in some RAMRIS scores correlated with later (weeks 24/28) changes in vdH-S. Significant correlations between RAMRIS change scores and clinical/radiographic change scores were weak. Conclusions MRI and clinical/laboratory/radiographic measures generally correlated well. Associations between earlier changes in CRP and later changes in RAMRIS synovitis/osteitis were observed. Changes in $\mathrm{MRI}$ and clinical/radiographic measures did not correlate well, probably because MRI is more sensitive than radiographs and more objective than DAS28-CRP.

MRI is more sensitive than radiographs in detecting joint erosions ${ }^{1-6}$ in rheumatoid arthritis (RA). Unlike radiographs, MRI can detect synovitis and bone marrow oedema, pre-erosive inflammatory changes that increase the risk of new erosions. ${ }^{7-13}$ Areas of bone appearing as osteitis/bone marrow oedema by MRI are heavily infiltrated by inflammatory cells and osteoclasts. ${ }^{14}$ The detection and treatment of pre-erosive inflammatory changes ${ }^{10} 15$ are crucial to limiting generally irreversible osseous joint damage. ${ }^{16}$

We have reported the results of radiographic and MRI assessments from two large phase III trials (GO-BEFORE, methotrexate-naive patients; ${ }^{17-19}$ GO-FORWARD, patients with inadequate response to methotrexate therapy) ${ }^{182021}$ that evaluated the efficacy of golimumab (a human monoclonal antibody to tumour necrosis factor alpha) in RA. MRI findings correlate with clinical, laboratory, imaging and histological measures of inflammation in RA. ${ }^{15} 16$ While MRI appears more sensitive than radiographs in detecting bone erosion, the ability of the RA MRI scoring (RAMRIS) system to detect erosive changes earlier/more often than the van der Heijde modification of the Sharp(vdH-S) scoring systems and the relationship between RAMRIS scores and laboratory/clinical measures of inflammation in large randomised clinical trials (eg, GO-BEFORE and GO-FORWARD MRI substudies) need to be assessed.

\section{PATIENTS AND METHODS}

Patients (318 GO-BEFORE, 240 GO-FORWARD) enrolled at willing and capable sites participated in MRI substudies. ${ }^{19} 21$ Disease activity was assessed using serum C-reactive protein (CRP) concentrations and 28-joint count disease activity score (DAS28) (calculated using CRP; DAS28 hereafter) scores. ${ }^{22}$ Structural damage (bone erosion, joint space narrowing) was measured using vdH-S scores. ${ }^{18} 23$ Preliminary assessments of relationships between RAMRIS synovitis, bone oedema (osteitis) and bone erosion scores and DAS28 scores, CRP levels and total vdH-S scores were accomplished by the determination of Spearman correlation coefficients $\left(r_{s}\right)$ for all treatment groups combined.

\section{RESULTS}

\section{Baseline patient characteristics}

Methotrexate-naive patients appeared to have more active inflammation but less structural damage than patients with an inadequate response to methotrexate (table 1).

\section{Cross-sectional data correlations}

\section{DAS28 versus RAMRIS scores}

In GO-BEFORE, significant $(\mathrm{p}<0.01)$ correlations were observed between baseline DAS28 scores and baseline RAMRIS synovitis $\left(r_{s}=0.40\right)$, bone oedema/ osteitis $\left(r_{s}=0.18\right)$, and bone erosion $\left(r_{s}=0.21\right)$ scores (table 2). Significant $(p<0.001)$ correlations were also observed between week 24 DAS28 scores and week 24 RAMRIS synovitis $\left(r_{s}=0.30\right)$, bone oedema/ osteitis $\left(r_{s}=0.22\right)$ and bone erosion $\left(r_{s}=0.23\right)$ scores Correlations in GO-FORWARD were weak. 
Table 1 Baseline clinical characteristics of the GO-BEFORE and GO-FORWARD MRI substudy populations

\begin{tabular}{|c|c|c|}
\hline \multirow[b]{2}{*}{ Characteristic } & \multicolumn{2}{|c|}{ All MRI substudy patients } \\
\hline & $\begin{array}{l}\text { GO-BEFORE } \\
\text { (methotrexate-naive) }\end{array}$ & $\begin{array}{l}\text { GO-FORWARD } \\
\text { (methotrexate } \\
\text { inadequate response) }\end{array}$ \\
\hline $\begin{array}{l}\text { Patients randomly assigned } \\
\text { to treatment, } n\end{array}$ & 318 & 240 \\
\hline \multirow[t]{2}{*}{ Women, n (\%) } & $257(80.8 \%)$ & $200(83.3 \%)$ \\
\hline & \multicolumn{2}{|c|}{ Median (IQR) } \\
\hline Age (years) & $50.0(41.0-58.0)$ & $51.0(43.0-58.0)$ \\
\hline Disease duration (years) & $1.2(0.6-3.7)$ & $6.3(3.0-13.5)$ \\
\hline Swollen joints (0-66) & $10.0(7.0-16.0)$ & $10.0(7.0-18.0)$ \\
\hline Tender joints (0-68) & $23.5(13.0-35.0)$ & $21.0(11.0-31.0)$ \\
\hline $\mathrm{CRP}(\mathrm{mg} / \mathrm{dl})$ & $1.2(0.5-2.7)$ & $0.8(0.4-2.0)$ \\
\hline $\mathrm{ESR}(\mathrm{mm} / \mathrm{h})$ & $38.0(22.0-58.0)$ & $36.0(22.0-50.0)$ \\
\hline \multirow[t]{3}{*}{ DAS28 score (0-10) } & $5.5(4.8-6.3)$ & $5.3(4.5-6.03)$ \\
\hline & \multicolumn{2}{|c|}{ Mean \pm SD } \\
\hline & \multicolumn{2}{|c|}{ Median (IQR) } \\
\hline Total vdH-S score (0-448) & $\begin{array}{l}20.5 \pm 38.1 \\
5.5(2.0-21.5)\end{array}$ & $\begin{array}{l}36.2 \pm 46.8 \\
15.8(2.5-50.8)\end{array}$ \\
\hline \multicolumn{3}{|l|}{ RAMRIS scores } \\
\hline $\begin{array}{l}\text { Synovitis, wrist plus } \\
\operatorname{MCP}(0-21)^{*}\end{array}$ & $\begin{array}{l}9.5 \pm 5.0 \\
9.5(5.5-13.5)\end{array}$ & $\begin{array}{l}7.0 \pm 4.3 \\
7.0(3.5-9.5)\end{array}$ \\
\hline Bone oedema/osteitis (0-69) & $\begin{array}{l}10.0 \pm 10.0 \\
6.5(2.5-15.5)\end{array}$ & $\begin{array}{l}6.9 \pm 9.1 \\
2.0(0.0-10.7)\end{array}$ \\
\hline Bone erosion $(0-230)$ & $\begin{array}{l}21.2 \pm 23.7 \\
14.5(10.0-22.5)\end{array}$ & $\begin{array}{l}24.4 \pm 28.1 \\
13.9(6.5-29.5)\end{array}$ \\
\hline
\end{tabular}

\footnotetext{
Data are presented for all treatment groups combined.

* Several sites did not have the capability to obtain postgadolinium images of both the wrist and the metacarpophalangeal joints; therefore, RAMRIS synovitis scores are summarised and assessed for the subgroups of patients with both determinations. CRP, C-reactive protein; DAS28, 28-joint disease activity score calculated using CRP; ESR, erythrocyte sedimentation rate; MCP, metacarpophalangeal; RAMRIS, rheumatoid arthritis MRI scoring system; vdH-S, van der Heijde modified Sharp score.
}

Serum CRP concentration versus RAMRIS scores

In GO-BEFORE, significant $(\mathrm{p}<0.001)$ correlations were observed between baseline CRP concentrations and baseline RAMRIS synovitis $\left(r_{s}=0.36\right)$, bone oedema/osteitis $\left(r_{s}=0.37\right)$ and bone erosion $\left(r_{\mathrm{s}}=0.30\right)$ scores. Significant but weaker correlations were observed between week 24 CRP concentrations and week 24 RAMRIS scores. Correlations observed in GO-FORWARD were weak.

\section{vdH-S versus RAMRIS scores}

In GO-BEFORE, significant $(\mathrm{p}<0.001)$ correlations were observed between baseline total vdH-S and baseline RAMRIS synovitis $\left(r_{s}=0.26\right)$, bone oedema/osteitis $\left(r_{s}=0.49\right)$ and bone erosion $\left(r_{s}=0.64\right.$; figure $\left.1 \mathrm{~A}\right)$ scores. Similar significant correlations were observed between week 28 total vdH-S and week 24 RAMRIS scores (figure 1B). Correlations between vdH-S and RAMRIS erosion scores were significant $(p<0.001)$ and strong at baseline $\left(r_{\mathrm{s}}=0.58\right)$ and weeks $24 / 28\left(r_{\mathrm{s}}=0.59\right)$.

In GO-FORWARD, significant $(\mathrm{p}<0.001)$ correlations were observed between total vdH-S and RAMRIS synovitis $\left(\mathrm{r}_{\mathrm{s}}=0.28\right)$, bone oedema/osteitis $\left(r_{s}=0.53\right)$ and bone erosion $\left(r_{s}=0.77\right)$ baseline scores. Findings were consistent at week 24 . Correlations between vdH-S and RAMRIS erosion scores were significant $(p<0.001)$ and strong at baseline $\left(r_{s}=0.73\right)$ and week $24\left(r_{s}=0.71\right)$.

\section{Change score correlations}

DAS28 versus RAMRIS change scores

Changes from baseline to week 12 and week 24 in DAS28 scores paralleled changes from baseline to week 12 and week 24 , respectively, in each RAMRIS score for GO-BEFORE and for RAMRIS bone oedema/osteitis scores only for GO-FORWARD (table 2). The association between RAMRIS change scores at week 12 and later changes in clinical response (week 24 DAS28) was significant only for the RAMRIS synovitis score in GO-BEFORE and

Table 2 Spearman correlation coefficients and $p$ values for the relationship between RAMRIS scores and clinical, laboratory and radiographic findings

\begin{tabular}{|c|c|c|c|c|c|c|}
\hline & \multicolumn{3}{|c|}{ GO-BEFORE (methotrexate-naive) } & \multicolumn{3}{|c|}{ G0-FORWARD (methotrexate inadequate response) } \\
\hline Baseline DAS28 & $0.40(p<0.001)$ & $0.18(p=0.002)$ & $0.21(p<0.001)$ & $0.17(p=0.021)$ & $0.00(p=0.96)$ & $-0.02(p=0.741)$ \\
\hline Baseline CRP & $0.36(p<0.001)$ & $0.37(p<0.001)$ & $0.30(p<0.001)$ & $0.27(p<0.001)$ & $0.21(p=0.002)$ & $0.13(p=0.065)$ \\
\hline Baseline total vdH-S & $0.26(p<0.001)$ & $0.49(p<0.001)$ & $0.64(p<0.001)$ & $0.28(p<0.001)$ & $0.53(p<0.001)$ & $0.77(p<0.001)$ \\
\hline Week 24 DAS28 & $0.30(p<0.001)$ & $0.22(p<0.001)$ & $0.23(p<0.001)$ & $0.15(p=0.05)$ & $0.00(p=0.96)$ & $0.01(p=0.89)$ \\
\hline Week 24 CRP & $0.24(p<0.001)$ & $0.25(p<0.001)$ & $0.23(p<0.001)$ & $0.21(p=0.009)$ & $0.02(p=0.84)$ & $-0.02(p=0.83)$ \\
\hline Weeks $24 / 28$ total vdH-S & $0.25(p<0.001)$ & $0.48(p<0.001)$ & $0.65(p<0.001)$ & $0.47(p<0.001)$ & $0.54(p<0.001)$ & $0.76(p<0.001)$ \\
\hline Weeks $24 / 28 \mathrm{vdH}-\mathrm{S}$ erosion score & - & - & $0.59(p<0.001)$ & - & - & $0.71(p<0.001)$ \\
\hline \multicolumn{7}{|l|}{ RAMRIS $\Delta$ to week 12 vs: } \\
\hline DAS28 $\Delta$ to week 12 & $0.21(p=0.001)$ & $0.17(p=0.008)$ & $0.14(p=0.028)$ & $0.14(p=0.08)$ & $0.21(p=0.004)$ & $0.01(p=0.87)$ \\
\hline vdH-S erosion score $\Delta$ to weeks $24 / 28$ & - & - & $0.03(p=0.59)$ & - & - & $-0.15(p=0.07)$ \\
\hline \multicolumn{7}{|l|}{ RAMRIS $\Delta$ to week 24 vs: } \\
\hline DAS28 $\Delta$ to week 24 & $0.22(p<0.001)$ & $0.13(p=0.036)$ & $0.17(p=0.006)$ & $0.36(p<0.001)$ & $0.21(p=0.008)$ & $0.10(p=0.22)$ \\
\hline CRP $\% \Delta$ to week 24 & $-0.20(p=0.002)$ & $-0.25(p<0.001)$ & $-0.06(p=0.34)$ & $-0.32(p<0.001)$ & $-0.22(p=0.007)$ & $-0.03(p=0.69)$ \\
\hline Total vdH-S $\Delta$ to weeks $24 / 28$ & $0.13(p=0.06)$ & $0.07(p=0.31)$ & $0.03(p=0.63)$ & $0.12(p=0.22)$ & $0.16(p=0.09)$ & $-0.06(p=0.50)$ \\
\hline vdH-S erosion score $\Delta$ to weeks $24 / 28$ & - & - & $0.07(p=0.28)$ & - & - & $-0.03(p=0.75)$ \\
\hline
\end{tabular}

\footnotetext{
Data are presented for all treatment groups combined.
}

*Several sites did not have the capability to obtain postgadolinium images of both the wrist and the metacarpophalangeal joints; therefore, RAMRIS synovitis scores are summarised and assessed for the subgroups of patients with both determinations.

CRP, C-reactive protein; DAS28, 28-joint disease activity score calculated using CRP; RAMRIS, rheumatoid arthritis MRI score; vdH-S, van der Heijde modified Sharp score. 

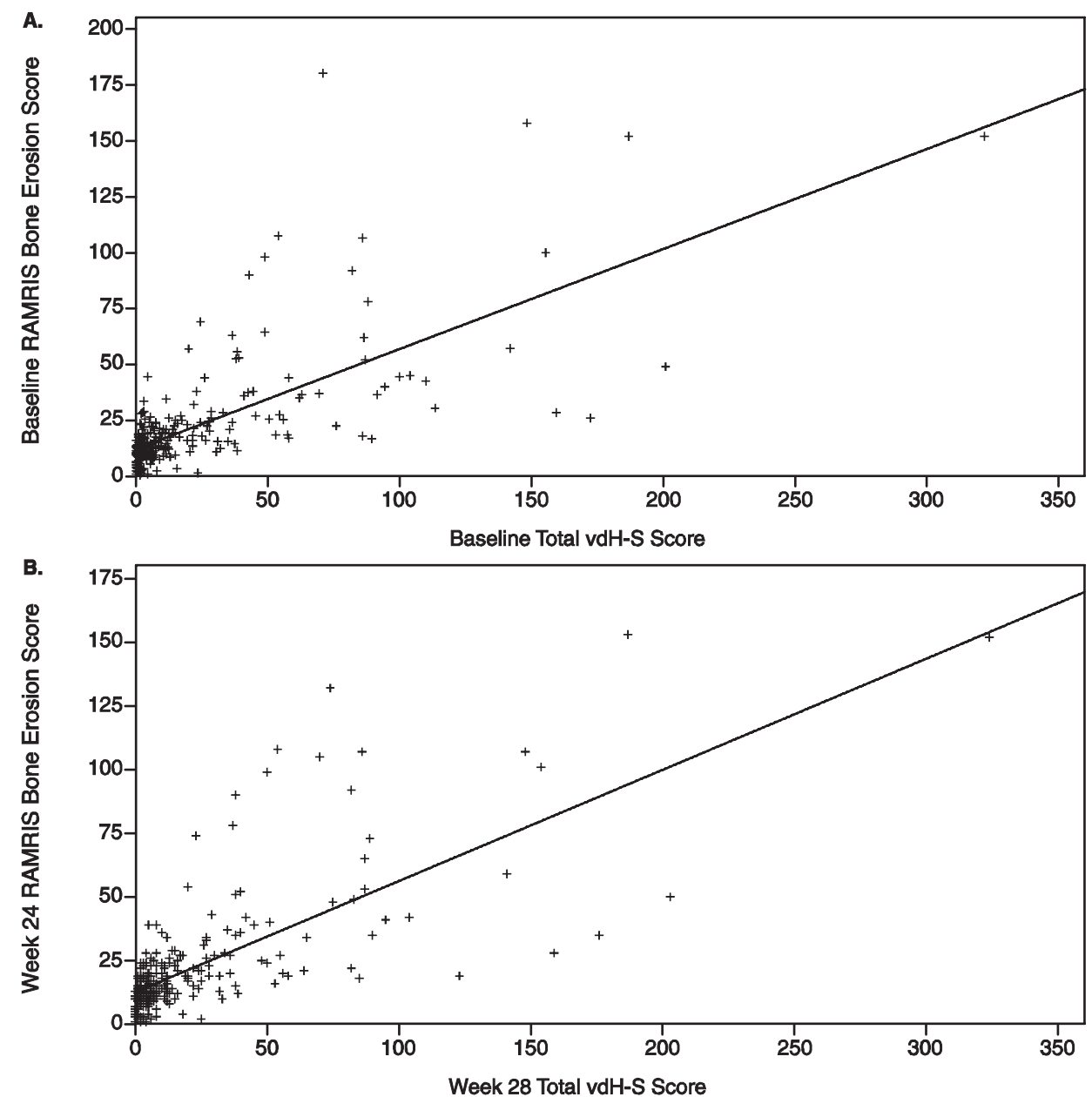

c.

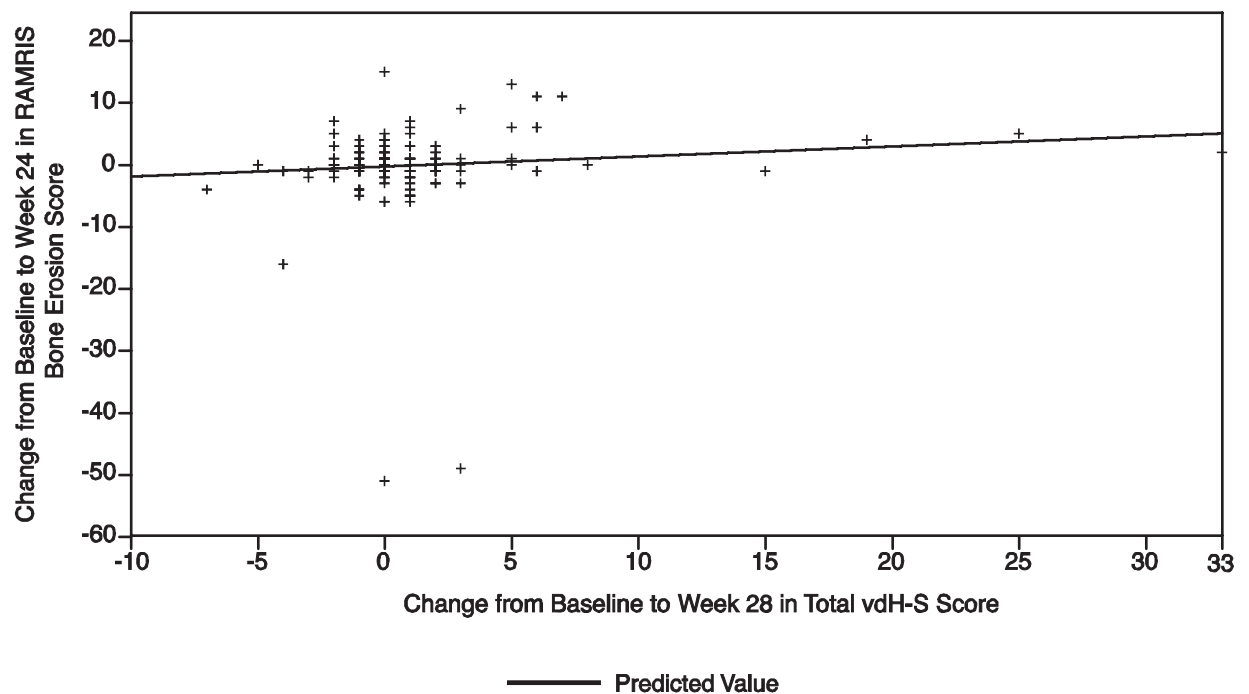

Figure 1 Relationship between Rheumatoid Arthritis MRI Scoring (RAMRIS) system bone erosion scores and total van der Heijde-modified Sharp (vdH-S) scores for absolute scores at baseline (A), absolute scores at week 24/28 (B), and changes in scores from baseline to week 24/28 (C) in the GO-BEFORE study. Observed data are presented for all treatment groups combined.

for synovitis and bone oedema/osteitis, but not bone erosion, RAMRIS scores in GO-FORWARD.

\section{Serum CRP concentration versus RAMRIS change scores}

Changes from baseline to week 12 and week 24 in serum CRP concentrations paralleled RAMRIS synovitis and bone oedema/osteitis, but not bone erosion, change scores. Week 4 (an early time point) CRP change was associated with later changes at week 12 in synovitis and bone oedema/osteitis, but not bone erosion, RAMRIS scores.

vdH-S versus RAMRIS change scores

In GO-BEFORE, changes from baseline to week 12 and week 24 in RAMRIS scores generally did not correlate with changes 
from baseline to week 28 in total vdH-S score (figure 1C), with the exception of a statistically significant but weak correlation between week 12 RAMRIS bone oedema/osteitis and week 28 total vdH-S change scores $\left(\mathrm{r}_{\mathrm{s}}=0.14, \mathrm{p}=0.033\right)$. In GO-FORWARD, week 24 RAMRIS change scores did not correlate with week 24 total vdH-S change scores. However, week 12 RAMRIS bone erosion changes weakly predicted later changes at week 24 in total vdH-S scores $\left(r_{s}=-0.18, p=0.027\right)$. Correlations between $\mathrm{vdH}-\mathrm{S}$ erosion and RAMRIS erosion change scores were not significant in either study.

\section{DISCUSSION}

Consistent with previous reports, ${ }^{15}{ }^{16}$ in our preliminary assessment of MRI data derived from the largest randomised, controlled trials evaluating RAMRIS scores in RA patients, we observed strong and significant correlations of cross-sectional data at baseline and week 24 between RAMRIS bone erosion and total vdH-S radiographic scores and between RAMRIS bone erosion and vdH-S erosion scores. At both baseline and week 24 of GO-BEFORE, statistically significant correlations were observed between each RAMRIS score and DAS28 scores and between each RAMRIS score and CRP concentrations. Overall, the correlations between RAMRIS scores and evaluated clinical/ laboratory/radiographic measures indicated that MRI findings represent disease activity and structural damage status as measured by conventional methods, thus confirming the findings of previously reported smaller studies and anecdotal reports. ${ }^{15} 16$

Interestingly, clinical/radiographic change scores generally did not correlate well with RAMRIS change scores. Changes in CRP, however, did correlate well with changes in RAMRIS measures of inflammation (synovitis and bone oedema/osteitis). In particular, early (week 4) changes in CRP may predict future changes in RAMRIS scores. The relatively stronger correlation observed between CRP and MRI change scores could be due to the objectivity of these measures. The DAS28 change score (clinical measure of disease activity) generally did not correlate well with RAMRIS change scores, perhaps because of the composite nature of the DAS28, which includes the subjective tender joint count not measured by MRI. In particular, if tenderness is due to factors beyond inflammation (eg, higher pain perception of the patient, fibromyalgia, etc), the DAS28 score may not correlate with RAMRIS measures of inflammation depending on the relative contribution of the tender joint count to the DAS28 score. While persistently high DAS28 scores predispose patients to more structural damage progression, the actual degree of progression varies widely across patients. Furthermore, in GO-FORWARD both RAMRIS and radiographic structural damage progression were minimal in all treatment arms including the control arm. Given the wide variability in patients' responses (as measured by DAS28) to therapeutic interventions and that only a small proportion of patients showed structural progression, a much larger sample size (than was available in these substudies) may have been needed to study such correlations, or lack thereof, adequately in these RA assessment tools. We also conducted post-hoc analyses using only the control arm to eliminate the possible impact of golimumab treatment on change scores, and results were similar to those described above. However, the much smaller sample size (approximately 25\% less than analyses involving all treatment arms combined) in these analyses should be considered in interpreting these post-hoc results.

The lack of strong correlation between RAMRIS erosion and vdH-S change scores could be due to the higher sensitivity of MRI versus radiographs in detecting bone erosion. ${ }^{1-6} 19$
Anti-tumour necrosis factor agents dramatically inhibit radiographic progression; thus, the association between RAMRISdetected changes in structural damage and vdH-S scores may best be assessed in control groups with the possibility of further progression. This was not the case for GO-FORWARD, as minimal progression was observed in all patients regardless of treatment. ${ }^{18} 21$ The lack of strong concordance between RAMRIS bone erosion and vdH-S (total and erosion) change scores may also be related to RAMRIS measuring the wrist plus metacarpophalangeal joints of one hand, while the total vdH-S scoring incorporates joints of both hands and feet. As the total vdH-S score includes the joint space narrowing subscore, while the RAMRIS score does not, an analysis to evaluate the correlation of changes in the vdH-S erosion subscore only and in RAMRIS erosion scores was conducted; these results also did not indicate good correlation. Finally, wide individual variation was observed in change scores, again possibly implicating insufficient sample size and little radiographic or MRI-detectable progression of structural damage in GO-FORWARD. ${ }^{18} 21$ Interestingly, even though the MRI and clinical/radiographic change scores did not correlate well in either GO-BEFORE or GO-FORWARD, the overall results of these MRI substudies were consistent with radiographic findings in the overarching study populations.

Taken together, findings derived from GO-BEFORE and GO-FORWARD MRI substudies indicate that RAMRIS scores and clinical/laboratory/radiographic measures at certain time points (ie, baseline, week 24) generally correlate well. For change scores, only changes in CRP correlated well with RAMRIS changes. The lack of strong/consistent correlations between vdH-S/DAS28 change scores and RAMRIS change scores is probably related to differential sensitivities of MRI and $\mathrm{x}$-ray for detecting erosive changes, the subjective component of DAS28, wide individual variations in reporting joint tenderness, and analysing all patients regardless of treatment group. Further in-depth analyses beyond this preliminary examination are underway to understand fully the relationship between MRI assessments and other measures of disease activity/progression of structural damage and the value of MRI in clinical practice and trials.

Acknowledgements The authors would like to thank the patients, investigators and study personnel who made these trials possible. The authors also thank Michelle Perate MS and Mary H Whitman PhD of Janssen Biotech, Inc., who helped prepare the manuscript.

Funding These studies were funded by Centocor Research and Development, Inc. and Schering-Plough Research Institute, Inc.

Competing interests PE has received consulting fees, speaking fees and/or research grants from Abbott Laboratories, Bristol-Myers Squibb, Centocor, Roche, Pfizer, UCB and Merck, Sharpe and Dohme. DvdH has received consulting fees, speaking fees and/or honoraria from Abbott, Amgen, AstraZeneca, Bristol-Myers Squibb, Centocor, Chugai, Eli Lilly, GlaxoSmithKline, Merck, Novartis, Otsuka, Pfizer, Roche, Sanofi-Aventis, Schering-Plough, UCB and Wyeth. MØ has received consulting fees, speaking fees and/or research grants from Abbott Laboratories, Amgen Inc., Bristol-Myers Squibb, Centocor, F Hoffmann-LaRoche Ltd., Genmab, GlaxoSmithKline, Novartis Pharmaceuticals Corporation, Pfizer Pharmaceuticals, Schering-Plough Corporation, UCB and Wyeth Pharmaceuticals. PGC has received consulting fees, speaking fees and/or research grants from Astra Zeneca, Bristol-Myers Squibb, Centocor, Merck, Sharpe and Dohme, Novartis, Roche and Pfizer. MCG has received grant support from, and served as a consultant to, Johnson \& Johnson, Inc., New Brunswick, New Jersey. ECK has received consulting fees, speaking fees and/or research grants from Abbott Laboratories, Amgen Inc., AstraZeneca Pharmaceuticals LP, Bristol-Myers Squibb, Centocor, F Hoffmann-LaRoche Ltd., Genentech Inc., Novartis Pharmaceuticals Corporation, Pfizer Pharmaceuticals, Schering-Plough Corporation, UCB and Wyeth Pharmaceuticals. RF has received consulting fees and/ or research grants from Abbott Laboratories, Amgen Inc., Bristol-Myers Squibb, Centocor, F Hoffmann-LaRoche Ltd., GlaxoSmithKline, Novartis Pharmaceuticals Corporation, Pfizer Pharmaceuticals, UCB, Genentech, Lexicon, Lily and Wyeth Pharmaceuticals. ECH, WX and SX are employees of Centocor, a wholly owned 
subsidiary of Johnson \& Johnson, Inc. (J\&J) and own stock in J\&J. MUR was formerly (during the conduct of this study) employed by Centocor. He is currently employed by Pfizer, Inc. and owns J\&J and Pfizer stocks.

Provenance and peer review Not commissioned; externally peer reviewed.

\section{REFERENCES}

1. Peterfy CG. New developments in imaging in rheumatoid arthritis. Curr Opin Rheumatol 2003;15:288-95.

2. Hoving JL, Buchbinder R, Hall S, et al. A comparison of magnetic resonance imaging, sonography, and radiography of the hand in patients with early rheumatoid arthritis. J Rheumatol 2004;31:663-75.

3. Klarlund M, Ostergaard M, Jensen KE, et al. Magnetic resonance imaging, radiography, and scintigraphy of the finger joints: one year follow up of patients with early arthritis. The TIRA Group. Ann Rheum Dis 2000;59:521-8.

4. Østergaard M, Hansen M, Stoltenberg M, et al. New radiographic bone erosions in the wrists of patients with rheumatoid arthritis are detectable with magnetic resonance imaging a median of two years earlier. Arthritis Rheum 2003:48:2128-31.

5. Østergaard M, Pedersen SJ, Døhn UM. Imaging in rheumatoid arthritis - status and recent advances for magnetic resonance imaging, ultrasonography, computed tomography and conventional radiography. Best Pract Res Clin Rheumatol 2008:22:1019-44.

6. Mundwiler ML, Maranian P, Brown DH, et al. The utility of MRI in predicting radiographic erosions in the metatarsophalangeal joints of the rheumatoid foot: a prospective longitudinal cohort study. Arthritis Res Ther 2009;11:R94. Comment in Arthritis Res Ther 2009;11:124.

7. Conaghan PG, O'Connor P, McGonagle D, et al. Elucidation of the relationship between synovitis and bone damage: a randomized magnetic resonance imaging study of individual joints in patients with early rheumatoid arthritis. Arthritis Rheum 2003;48:64-71.

8. Huang J, Stewart N, Crabbe J, et al. A 1-year follow-up study of dynamic magnetic resonance imaging in early rheumatoid arthritis reveals synovitis to be increased in shared epitope-positive patients and predictive of erosions at 1 year. Rheumatology (Oxford) 2000;39:407-16.

9. McQueen FM, Benton N, Perry D, et al. Bone edema scored on magnetic resonance imaging scans of the dominant carpus at presentation predicts radiographic joint damage of the hands and feet six years later in patients with rheumatoid arthritis. Arthritis Rheum 2003;48:1814-27.

10. Bøyesen $\mathbf{P}$, Haavardsholm EA, Østergaard M, et al. MRI in early rheumatoid arthritis: synovitis and bone marrow oedema are independent predictors of subsequent radiographic progression. Ann Rheum Dis 2011;70:428-33.
11. McQueen FM, Ostendorf $\mathrm{B}$. What is MRI bone oedema in rheumatoid arthritis and why does it matter? Arthritis Res Ther 2006;8:222

12. Jimenez-Boj E, Nöbauer-Huhmann I, Hanslik-Schnabel B, et al. Bone erosions and bone marrow edema as defined by magnetic resonance imaging reflect true bone marrow inflammation in rheumatoid arthritis. Arthritis Rheum 2007:56:1118-24.

13. Schett G. Bone marrow edema. Ann NY Acad Sci 2009;1154:35-40.

14. Dalbeth N, Smith T, Gray S, et al. Cellular characterisation of magnetic resonance imaging bone oedema in rheumatoid arthritis; implications for pathogenesis of erosive disease. Ann Rheum Dis 2009;68:279-82.

15. Hodgson RJ, O'Connor P, Moots R. MRI of rheumatoid arthritis image quantitation for the assessment of disease activity, progression and response to therapy. Rheumatology (Oxford) 2008;47:13-21.

16. Giovagnoni A, Valeri G, Burroni E, et al. Rheumatoid arthritis: follow-up and response to treatment. Eur J Radiol 1998:27(Suppl 1):S25-30.

17. Emery P, Fleischmann RM, Moreland LW, et al. Golimumab, a human anti-tumor necrosis factor alpha monoclonal antibody, injected subcutaneously every four weeks in methotrexate-naive patients with active rheumatoid arthritis: twenty-four-week results of a phase III, multicenter, randomized, double-blind, placebo-controlled study of golimumab before methotrexate as first-line therapy for early-onset rheumatoid arthritis. Arthritis Rheum 2009;60:2272-83.

18. Emery $\mathbf{P}$, Fleischmann $\mathrm{R}$, van der Heijde D, et al. The effects of golimumab on radiographic progression in rheumatoid arthritis: results of randomized controlled studies of golimumab before methotrexate therapy and golimumab after methotrexate therapy. Arthritis Rheum 2011;63:1200-10.

19. Østergaard M, Emery P, Conaghan PG, et al. Golimumab and methotrexate combination therapy significantly improves synovitis, osteitis and bone erosion compared with methotrexate alone - a magnetic resonance imaging study of 318 methotrexate-naïve rheumatoid arthritis patients. Arthritis Rheum; In press.

20. Keystone EC, Genovese MC, Klareskog L, et al. Golimumab, a human antibody to tumour necrosis factor $\{$ alpha\} given by monthly subcutaneous injections, in active rheumatoid arthritis despite methotrexate therapy: the GO-FORWARD Study. Ann Rheum Dis 2009;68:789-96.

21. Conaghan PG, Emery P, Østergaard M, et al. Assessment of inflammation and damage by MRI in established RA patients with methotrexate inadequate response receiving golimumab: results of the GO-FORWARD trial. Ann Rheum Dis 2011 Jul 21. Epub ahead of print doi:10.1136/ard.2010.146068.

22. Prevoo ML, van 't Hof MA, Kuper HH, et al. Modified disease activity scores that include twenty-eight-joint counts. Development and validation in a prospective longitudinal study of patients with rheumatoid arthritis. Arthritis Rheum 1995;38:44-8.

23. van der Heijde DM, van Riel PL, Nuver-Zwart IH, et al. Effects of hydroxychloroquine and sulphasalazine on progression of joint damage in rheumatoid arthritis. Lancet 1989;1:1036-8 